\title{
Deteriorated gene expression of selected calcium transporters in streptozotocin-induced diabetic hearts of Wistar rats
}

Comenius University in Bratislava, Faculty of Pharmacy, Department of Pharmacology and

Toxicology, Bratislava, Slovak Republic

Received 28 February, 2018, accepted 4 June, 2018

Abstract Aim: The aim is to identify the possible changes in the expression of genes, that regulate calcium homeostasis in cardiomyocytes in diabetes mellitus.

Methods: Male Wistar rats were randomized into two experimental protocols: short-term 5-days streptozotocin-induced diabetes protocol with 20 weeks old animals at the end of the protocol (total $\mathrm{N}=20$ ) and long-term 4-weeks protocol with 18 weeks of age at the end of the protocol (total $\mathrm{N}=38$ ). $50 \mathrm{mg} / \mathrm{kg}$ of streptozotocin (STZ) was administered in both protocols by a single intraperitoneal injection in $0,1 \mathrm{M}$ citrate buffer $(\mathrm{pH}=4.5)$. Control group (CON) received only vehiculum. Gene expressions in samples of left heart ventricle were measured by RT-qPCR method.

Results: The expression of SERCA2a in short-term protocol was decreased. In long-term protocol, decreased SERCA2a, TRPC4 and TRPC6 mRNA levels were observed ( $\left.{ }^{*} p<0.05\right)$. SERCA2a and TRPC4 mRNA levels exhibited statistical monotonic correlation in STZ-treated group in long-term protocol.

Conclusions: In diabetes mellitus, the calcium homeostasis in cardiomyocytes is altered and there could be a relation between alteration of internal sarcoplasmatic stores and store-operated calcium entry.

Keywords TRPC-diabetes-SERCA2a-calcium-heart ventricles

\section{INTRODUCTION}

Diabetic cardiomyopathy was previously associated with altered calcium handling. Decreased sarcoplasmatic load and sarco/endoplasmatic calcium ATPase (SERCA2a) activity contribute to intracellular calcium overload, that is associated with excitation-contraction coupling and other deterioration of cardiac function in diabetic models (Turan and Dhalla, 2014). TRPC (Transient receptor potential - canonical) channels are non-voltage-gated calcium channels that could be activated by intracellular calcium stores and take a part in the development of hypertrophy and heart failure (Watanabe et al., 2008). However, little is known about the contribution of cardiac TRPC channels in diabetes mellitus.

\section{METHODS}

\section{Animals and experimental design}

All the experimental procedures involving the use of experimental animals were approved by the State Veterinary and Food Administration of the Slovak Republic and by the
Ethics Committee of the Faculty of Pharmacy, Comenius University in Bratislava. All the animals were kept under standard conditions and received standardized rodent chow and water ad libitum. Male Wistar rats (Department of Toxicology and Laboratory Animal breeding, Dobra Voda, Slovak Republic) were randomized into two experimental protocols: short-term 5 days streptozotocin-induced diabetes with animals 20 weeks of age at the end of the protocol (total $\mathrm{N}=20$ ) and long-term 4 weeks streptozotocin-induced diabetes with animals 18 weeks old at the end of protocol (total $\mathrm{N}=38$ ). $50 \mathrm{mg} / \mathrm{kg}$ of streptozotocin (STZ; Chemos CZ s.r.o., Czech Republic) was administered in both protocols by a single intraperitoneal injection in $0,1 \mathrm{M}$ citrate buffer $(\mathrm{pH}=$ 4.5). Control group (CON) received only vehiculum.

\section{Glucose measurements and termination}

In short term 5 days protocol, the postprandial glucose blood levels were measured from inferior vena cava, post mortem. In long term 4 weeks protocol, the standard rat laboratory chow

\footnotetext{
*E-mail:mato.dragun@gmail.com
}

(c) European Pharmaceutical Journal 
was removed 12 hours before blood glucose measurement on the last day of experimental protocol. Water was provided ad libitum the whole time. Blood glucose levels were measured with point of care glucose testing device (Accutrend Plus system; Cobas/Roche Diagnostics International, Switzerland) from lateral tail vein after tail nicking. After the experimental protocol, animals were sacrificed by asphyxiation in carbon dioxide and samples of the left ventricular free-wall were dissected for RNA isolation.

\section{RNA isolation and RT-PCR}

Total RNA was isolated from left ventricular samples using Tri-Reagent $^{\circ}$ (Sigma-Aldrich, USA). The quality of isolated RNA was verified with agarose gel electrophoresis and microspectrophotometric analysis (NanoDropND-1000, Thermo Scientific, USA). Reverse transcription was performed using the High-Capacity cDNA KIT with RNAse inhibitor (Applied Biosystems, USA). Real-time polymerase chain reaction (PCR) was performed using SYBR Green detection (SYBR Select Master Mix, Life Technologies, USA) on StepOnePlusÔ Real-Time PCR System (Life Technologies, USA) according to the manufacturer's instructions. Expressions of natriuretic peptide A (ANP), sarco/endoplasmatic reticulum calcium ATPase 2a subunit (SERCA2a) and transient receptor potential canonical 4 and 6 (TRPC4, TRPC6) were evaluated using gene-specific primers. The results were normalized to the expressions of endogenous reference genes (Beta-2 microglobulin, B2M; hypoxanthine phosphoribosyltransferase 1, HPRT1). The following primers sequence were used: HPRT1 (forward: 'CAGCTTCCTCCTCAGACCGCTTT', reverse: 'TCACTAATCACGACGCTGGGACTG'), B2M (forward: 'ATGGAGCTCTGAATCATCTGG', reverse: 'AGAAGATGGTGTGCTCATTGC'), ANP (forward: 'GGGGGTAGGATTGACAGGAT', reverse: 'GGATCTTTTGCGATCTGCTC'), SERCA2a (forward: 'CCCGAAACTACCTGGAGCCTGCA', reverse: 'ATGCACGCACCCGAACACCC'), $\quad$ TRPC4 (forward: 'AGCCCAGCGGAGAGAAGCAG', reverse: 'GGTCTGGGCACCGAGACACC'), TRPC6 (forward: 'TCGTGGCGCATCCGAACTGC', reverse: GGAGGAGCTTGGTGCCTTCAAATCT'). All primers (SigmaAldrich, USA) were verified to yield a single PCR product with the correct molecular weight, and the absence of signal was verified when reverse transcription was omitted.

\section{Computational and statistical analysis}

Mean PCR efficiency estimates (E) per amplicon and quantification cycle $(\mathrm{Cq})$ values per sample were determined with LinRegPCR software (version 2015.0) and efficiency corrected relative expression ratios were calculated (all reactions had $\mathrm{E}>1.8$ and $\mathrm{Cq}<35$ ). All results were reported as mean \pm standard error of mean (SEM). Comparisons between
Table 1. Relative mRNA levels of ANP, SERCA2a, TRPC4 and TRPC6 genes in left ventricle samples divided in control group (CON) and group with streptozotocin-induced diabetes (STZ). Values are mean \pm SEM. $\left({ }^{*} p<0.05\right.$ vs. CON).

\begin{tabular}{|c|c|c|c|c|}
\hline \multirow{2}{*}{$\begin{array}{c}\text { Target } \\
\text { gene }\end{array}$} & \multicolumn{2}{|c|}{5 days protocol } & \multicolumn{2}{c|}{4 weeks protocol } \\
\cline { 2 - 5 } & $\begin{array}{c}\text { CON 5D } \\
(\mathrm{n}=10)\end{array}$ & $\begin{array}{c}\text { STZ 5D } \\
(\mathrm{n}=10)\end{array}$ & $\begin{array}{c}\text { CON 4W } \\
(\mathrm{n}=16)\end{array}$ & $\begin{array}{c}\text { STZ 4W } \\
(\mathrm{n}=12)\end{array}$ \\
\hline ANP & $1.00 \pm 0.09$ & $0.97 \pm 0.21$ & $1.00 \pm 0.01$ & $4.36 \pm 0.51^{*}$ \\
\hline SERCA2a & $1.00 \pm 0.06$ & $0.67 \pm 0.03^{*}$ & $1.00 \pm 0.04$ & $0.68 \pm 0.05^{*}$ \\
\hline TRPC4 & $1.00 \pm 0.16$ & $0.77 \pm 0.10$ & $1.00 \pm 0.09$ & $0.58 \pm 0.11^{*}$ \\
\hline TRPC6 & $1.00 \pm 0.09$ & $0.87 \pm 0.06$ & $1.00 \pm 0.08$ & $0.65 \pm 0.05^{*}$ \\
\hline
\end{tabular}

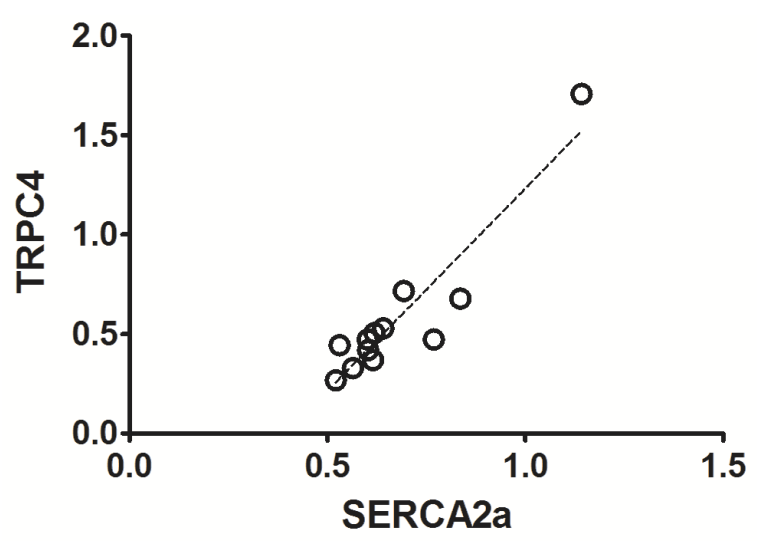

Figure 1. Association between MRNA levels of SERCA2a and TRPC4 in STZ-treated group after 4 weeks experimental diabetes. Spearman's rank correlation coefficient rho $=0.82\left({ }^{*} p<0.01\right)$.

the means of two groups were performed by Student's $t$ test for normally distributed data or Mann-Whitney test for non-parametric data, $p<0.05$ considered as statistically significant. Statistical dependence between gene expression values of the two genes was evaluated by the Spearman's rank correlation coefficient (rho). Data were handled by GraphPad Prism (GraphPad Software Inc., version 6).

\section{RESULTS}

Objective of our study was to monitor the development of changes in abundance of selected calcium transporters in a model of streptozotocin-induced simulation of type I diabetes mellitus from the short term 5 days protocol to a longer follow-up after 4 weeks from diabetes induction. Actual diabetes in treated animals was confirmed against the criterion of preprandial and postprandial blood glucose measurement $(\geq 7.0 \mathrm{mmol} / \mathrm{l}$ and $\geq 11.1 \mathrm{mmol} / \mathrm{l}$, resp.). 
Postprandial blood glucose levels in 5 days protocol were $21.39 \pm 1.55 \mathrm{mmol} / \mathrm{l}$ vs. $36.78 \pm 1.76 \mathrm{mml} / \mathrm{l}$ in control and STZ-treated group resp. ( $\left.{ }^{*} p<0.0001\right)$. Unusually, high levels in control group were the consequence of post mortem measurement after asphyxiation ion $\mathrm{CO}_{2}$ and probable adrenalin-induced release of glucose from liver. However, the difference between the groups is self-evident. Postprandial glycaemia was also higher in 4 weeks protocol: $13.92 \pm 2.74$ $\mathrm{mmol} / \mathrm{l}$ in STZ-treated group, vs. $6.19 \pm 0.50 \mathrm{mmol} / \mathrm{l}$ of CON (* $\mathrm{p}<0.05)$. Potential for diabetes related cardiac deterioration was estimated by myocardial expression of ANP, increased quantities were observed after 4 weeks, not in short term protocol. The expression of SERCA2a 5 days after STZ injection was decreased in contrast with other measured genes, where the expression levels remained unchanged. However, we found decreased mRNA levels of SERCA2a, with TRPC4 and TRPC6 after 4 weeks of experimental diabetes (Tab. 2). In addition, SERCA2a and TRPC 4 mRNA level exhibited statistical monotonic correlation in STZ-treated group in 4 weeks experimental protocol (Figure 1).

\section{DISCUSSION}

Several pathways or mechanisms contribute to the development of diabetic cardiomyopathy. One of the hypothesis is alteration in calcium homeostasis. We set up in our experimental design to assess the development of changes in mRNA expression of selected calcium handling transporters from short lasting streptozotocin-induced type 1 diabetes mellitus (DM) to a prolonged untreated diabetes. We observed, that the first changes in calcium transporter gene expression occur in short-term of DM, when the expression of SERCA2a, as one of the most important channel in intracellular calcium homeostasis, is downregulated and this downregulation is preserved also in long-term stages of DM. This decline of expression is in agreement with the findings of Zhao et al. (2014), who found out progressive decrease of SERCA2a expression during 12 weeks of experimental diabetes. Interestingly, decrease in SERCA2a expression preceded the increase of ANP expression, as a marker of nonspecific cardiac damage, that is present after 4 weeks of experimental diabetes. TRPC channels were previously associated with cardiac remodelling and the development of heart failure (Eder and Molkentin, 2011). We propose that TRPC4 and TRPC6 channels could also play a role in diabetic cardiomyopathy. We found both downregulated, however, only after 4 weeks of experimental diabetes. We also looked if mRNA expressions of TRPC4 channel, that is responsible for store-operated calcium entry and SERCA2a as a major regulator of sarcoplasmatic internal calcium stores, could correlate. We found that the expressions of these two agents significantly correlate. This suggested that the intracellular stores and store-operated calcium entry could be altered, and these two aspects could be related.

\section{ACKNOWLEDGEMENT}

This work was supported by grants: FAF UK/29/2017, VEGA 1/0304/17.

\section{References}

[1] Eder, P., Molkentin, J.D., 2011. TRPC Channels As Effectors of Cardiac Hypertrophy. Circ. Res. 108, 265-272. https://doi. org/10.1161/CIRCRESAHA.110.225888

[2] Turan, B., Dhalla, N.S. (Eds.), 2014. Diabetic Cardiomyopathy. Springer New York, New York, NY. https://doi.org/10.1007/978-14614-9317-4

[3] Watanabe, H., Murakami, M., Ohba, T., Takahashi, Y., Ito, H., 2008. TRP channel and cardiovascular disease. Pharmacol. Ther. 118, 337-351. https://doi.org/10.1016/j.pharmthera.2008.03.008

[4] Zhao, S.-M., Wang, Y.-L., Guo, C.-Y., Chen, J.-L., Wu, Y.-Q., 2014. Progressive decay of $\mathrm{Ca} 2+$ homeostasis in the development of diabetic cardiomyopathy. Cardiovasc. Diabetol. 13, 75. https:// doi.org/10.1186/1475-2840-13-75 